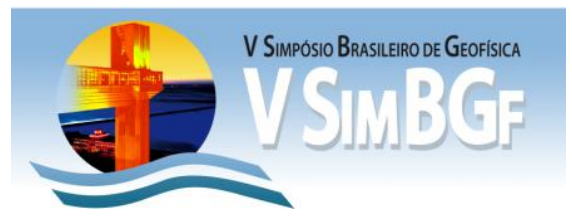

\title{
Migração pré-empilhamento Kirchhoff-feixes gaussianos 2,5D em verdadeira amplitude
} M. J. S. Costa ${ }^{* 1}$, G. Garabito ${ }^{2}$, João C. R. Cruz e Carlos A. S. Ferreira ${ }^{3}$

${ }^{1}$ Universidade Federal do Pará, ${ }^{2}$ Universidade Federal do Rio Grande do Norte e ${ }^{3}$ Agência Nacional do Petróleo

Copyright 2012, SBGf - Sociedade Brasileira de Geofísica

Este texto foi preparado para a apresentação no V Simpósio Brasileiro de Geofísica Salvador, 27 a 29 de novembro de 2012. Seu conteúdo foi revisado pelo Comitê Técnico do V SimBGt, mas não necessariamente representa a opinião da SBGf ou de seus associados. E proibida a reprodução total ou parcial deste material para propósitos comerciais sem prévia autorização da SBGt.

\begin{abstract}
An asymptotic procedure for computation of seismic wavefields in the paraxial vicinity of a central ray is proposed, based on the superposition of Gaussian Beams (GBs) and the Fresnel volume elements. GBs represent a regular and highly accurate description of the seismic wavefield, e.g., in singular regions of the propagating medium. Therefore, in this article we present a prestack depth migration in which the main mathematical operation is given by the superposition of GBs into the kernel of the 2.5D true-amplitude Kirchhoff prestack depth migration operator. Numerical examples show the influence on the final migrated images of the application of this operator when compared to the traditional Kirchhoff depth migration.
\end{abstract}

\section{Introdução}

Em todos os ambientes geológicos, dos mais simples aos mais complexos, a realização de um imageamento consiste em uma tarefa que apresenta três problemas fundamentais, quais sejam: a imagem se apresenta com baixa resolução, tem seu formato errôneo e se encontra posicionada no local errado. Assim, uma imagem interpretável significa obter-se uma energia sísmica focalizada e bem definida, com o formato correto e em sua posição verdadeira, isto é, quanto mais definida for à imagem e quanto mais verdadeira sua posição e formato, mais precisamente a estrutura poderá ser avaliada e explorada. Neste sentido, o método que define, modela e localiza a imagem denomina-se "migração", a qual representa uma das etapas mais importantes de todo processamento sísmico, em virtude da mesma transformar (migrar) os dados sísmicos em uma imagem da região de interesse em subsuperfície a ser interpretada. Existem diferentes tipos de migração e todas são consideradas como métodos no processo de imageamento, cada um com suas vantagens e desvantagens. Assim, a migração tem como objetivo principal, fornecer a imagem dos refletores de interesse em subsuperfície. Em virtude desta importância, ao longo dos anos foram desenvolvidos métodos de migração com o propósito de melhorar cada vez mais a resolução das imagens em subsuperfície. Dentre os métodos citados acima se destaca o método de migração tipo Kirchhoff (Schneider, 1978; Bleistein, 1987; Schleicher et al., 1993; Tygel et al., 1996), o qual é extensamente utilizado na industria de petróleo para obter imagens da subsuperfície a partir do dado sísmico. A migração tipo Kirchhoff é bastante versátil, visto permitir migrar dados completos ou apenas parte dele, no entanto, a referida migração faz um amplo uso da teoria do raio de ordem zero, como forma de encontrar a função de Green do problema de imageamento, sendo esta teoria aplicada de forma efetiva em meios suaves. Desta forma, alguns fenômenos de propagação da onda, como por exemplo, as difrações observadas em ambientes, onde a geologia do meio é complexa ou em meios que apresentam fortes variações laterais de velocidades, não podem ser simuladas de forma adequada. Somado a estes fatos, existem outras restrições vinculadas à teoria do raio de ordem zero, as quais se referem às singularidades ocasionais advindas da ocorrência de cáusticas (cruzamento de raios) ao longo do caminho de um determinado raio especular, das zonas de sombra, etc. Assim, havendo a necessidade crescente de melhorar a qualidade das imagens, estudiosos vem desenvolvendo pesquisas de imageamento sísmico (Albertin et al., 2004; Bleistein, 2008; Hill, 1990, 2001; Ferreira, 2007; Ferreira; Cruz, 2004a, 2005a, 2005b, 2005c, 2005d, 2009; Gray; Bleistein, 2009; Popov et al., 2010, Protasov; Tcheverda, 2011), pesquisas estas que se utilizam das vantagens do método de feixes gaussianos (do inglês Gaussian Beams ou GB's) na solução da equação da onda sísmica, tais vantagens sendo apresentadas em Cervený (1982, 1983, 1985, 2000), Popov(1982, 2002), Müller (1984), Novack (2003), Žácek (2006), Kravtsov et al., (2007)). Todas estas pesquisas estabelecem que a técnica de migração que utiliza os fundamentos teóricos dos GB's, torna-se uma técnica poderosa de imageamento sísmico, com precisão comparável às migrações que utilizam a solução da equação da onda, bem como, quando comparadas à flexibilidade e confiabilidade das migrações do tipo Kirchhoff. Diante do exposto, o presente trabalho abordará uma integral de migração denominada migração Kirchhoff 2,5D em verdadeira amplitude (Tygel et al., 1996), aplicada a uma classe de migração préempilhamento em profundidade. Contudo, tal integral será modificada em seu núcleo, através de uma aproximação do campo de ondas, definida por um operador integral de superposição de feixes gaussianos, como função de Green do problema de imageamento no domínio do afastamento fonte-receptor constante $(\mathrm{CO}$, do inglês common offset) similarmente a Ferreira e Cruz (2009). 


\section{Metodologia}

A forma geral da integral de migração do tipo Kirchhoff em 2,5D no domínio CO é dada por (Martins et al. 1997):

$I(R, t)=\frac{1}{\sqrt{2 \pi}} \int_{x_{m 1}}^{x_{m 2}} d x_{m} w_{2,5 D}\left(x_{m}, R\right) \partial_{t-}^{\frac{1}{2}} U\left(x_{m}, t=\tau_{D}\left(x_{m}, R\right)\right)$,

onde, $I(R, t=0)$ denota a amplitude migrada para um dado ponto $R$ da seção migrada, $U\left(x_{m}, t\right)$ representa o dado sísmico 2,5D na configuração $\mathrm{CO}$ a ser migrado, $\partial_{t-}^{\frac{1}{2}}$ indica uma operação matemática definida como uma meia derivada em relação ao tempo, $x_{m}$ sendo a coordenada do ponto médio, que neste caso também é a variável de integração. Neste sentido, variando-se os valores de $x_{m}$ são calculados os tempos de difração $\tau_{D}\left(x_{m}, R\right)$, para cada traço de entrada com seus respectivos pesos $w_{2,5 D}\left(x_{m}, R\right)$ para retirar das amplitudes advindas da migração 0 efeito do espalhamento geométrico. Assim, em todos os traços dentro da abertura $x_{m 1} \leq x_{m} \leq x_{m 2}$ são coletadas as amostras $U\left(x_{m}, t=\tau_{D}\left(x_{m}, R\right)\right)$ sobre a curva de difração aplicando-se os respectivos pesos, em seguida somamse todos os valores e o resultado acumulado $I(R)$ é colocado no ponto $R$ de saída. Portanto, ao percorrer todos os pontos $R$ do domínio migrado obtém-se a migração da seção sísmica de entrada. Agora, o operador de migração Kirchhoff 2,5D no domínio afastamento comum (Equação 1) será expresso pela seguinte representação matemática no domínio da frequência:

$$
I(R, \omega)=\sqrt{-\frac{i \omega}{2 \pi}} \int_{x_{m 1}}^{x_{m 2}} d x_{m} w_{2,5 D}\left(x_{m}, R\right)
$$

$\times \psi_{2,5}\left(x_{m}, \omega\right) e^{i \omega \tau_{D}\left(x_{m}, R\right)}$,

sendo o dado sísmico $\psi_{2,5}\left(x_{m}, \omega\right)$ determinado através de uma superposição de feixes gaussianos 2,5D (Costa, 2012), isto é:

$$
\psi_{2,5 D}\left(x_{m}, t\right)=\sqrt{\frac{-i \omega}{2 \pi}} \int_{x m_{1}^{P}}^{x_{m 2}^{P}} d x_{m}^{P} \sqrt{H_{11}}
$$

$\times D_{L}\left(x_{m}^{P}\right) \partial_{t-}^{\frac{1}{2}} U_{2,5 D}\left(x_{m}^{P}, t-T\left(x_{m}^{P}, x_{m}\right)\right)$,

com,

$T=p\left(x_{m}^{p}-x_{m}\right)+\frac{1}{2} H_{11}\left(x_{m}\right)\left(x_{m}^{p}-x_{m}\right)^{2}$,

onde, $T$ é o tempo de trânsito paraxial complexo, $U_{2,5 D} \circ$ dado de entrada, $\psi_{2,5}\left(x_{m}, \omega\right)$ valor do eventos empilhados com feixes gaussianos, $p$ indicando $o$ parâmetro vagarosidade do raio central, $H_{11}$ a zona de Fresnel projetada, $D_{L}$ a janela gaussiana, $L$ a meia largura do feixe e $x_{m}^{P}$ caracterizando a posição dentro da janela de empilhamento. Assim, considerando a Equação (2) e a definição da Equação (3), ambas no domínio da frequência, determina-se a integral de migração modificada Kirchhoff-feixes gaussianos 2,5D em verdadeira amplitude, como segue (Costa, 2012):

$$
\begin{aligned}
I(R, \omega) & =\sqrt{\frac{-i \omega}{2 \pi}} \int_{x_{m 1}}^{x_{m 2}} d x_{m} w_{2,5 D}\left(x_{m}, R\right) e^{i \omega \tau_{D}\left(x_{m}, R\right)} \\
& \times \sqrt{\frac{-i \omega}{2 \pi}} \int_{x_{m 1}^{P}}^{x_{m 2}^{P}} d x_{m}^{P} \sqrt{H_{11}} D_{L}\left(x_{m}^{P}\right) U_{2,5 D}\left(x_{m}^{P}, \omega\right)
\end{aligned}
$$

$\times e^{i \omega T\left(x_{m}^{p}, x_{m}\right)}$

O operador de migração acima tem as mesmas características que o operador de migração definido por Schleicher et al. (1993) ou em Tygel et al. (1996). Contudo, ao longo da curva de difração $\tau_{D}\left(x_{m}, R\right)$, contribuições paraxiais $T\left(x_{m}^{p}, x_{m}\right)$ de eventos difratados presentes em traços contidos no interior da zona de Fresnel considerada $x_{m 1}^{p} \leq x_{m}^{p} \leq x_{m 2}^{p}$, também contribuem para a amplitude dos eventos de reflexão observados em $x_{m}$ ao longo de sua própria curva de empilhamento $\tau_{D}\left(x_{m}, R\right)$ (Figura 1).

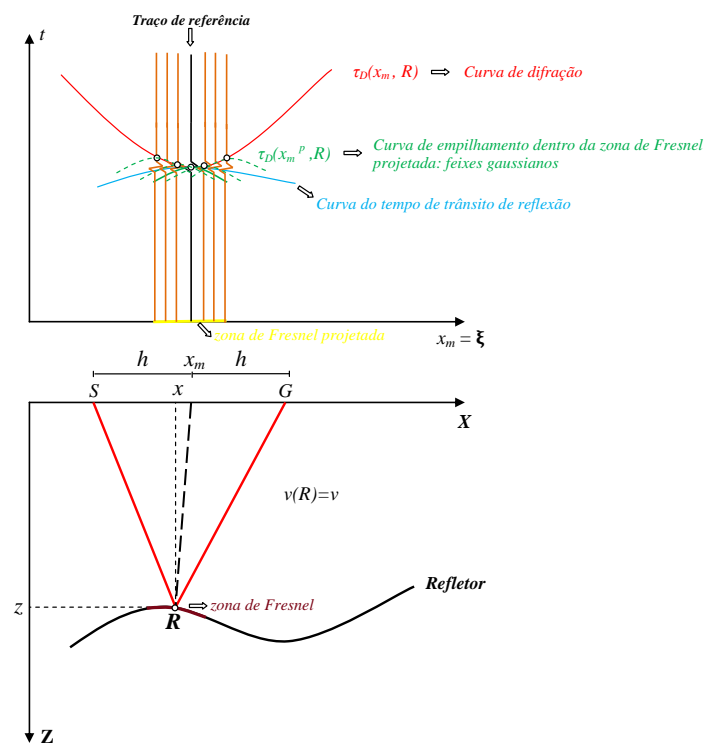

Figura 1- Interpretação geométrica da técnica de migração Kirchhoff modificada na configuração de medida afastamento comum em um meio homogêneo, onde $x_{m}$ é a coordenada do ponto médio entre fonte $S$ e geofone $G, h$ o meio afastamento entre fonte $S$ e geofone $G, x$ coordenada espacial do ponto $R$ no domínio migrado, $z$ a profundidade e $v$ a velocidade do meio.

As principais etapas do algoritmo de migração Kirchhofffeixes gaussianos no domínio CO são:

1. Entrada da seção CO;

2. Para cada ponto da seção de saída, calcular:

- ponto médio, meio afastamento, curva de difração, função peso associada à referida curva de difração;

\section{Tempos da curva de difração:}

-para cada tempo das curvas de difração, calcular a curva paraxial, os parâmetros que definem a abertura e o 
peso da integral de superposição dos feixes gaussianos, em seguida faz-se o empilhamento dos traços paraxiais;

\section{Somando amplitudes:}

- Somam-se as amplitudes advindas da soma paraxial ao longo da curva de difração, repetindo o procedimento para todos os pontos de amostragem da seção de saída;

\section{Saída:}

-Seção migrada final

\section{Resultados numéricos obtidos a partir de um dado sintético no domínio $\mathrm{CO}$}

Para efeito de ilustração, inicialmente será apresentado às respostas ao impulso dos operadores de migração Kirchhoff convencional (K) e Kirchhoff modificado (KGB) para um meio com gradiente constante, também apresentar-se-á uma comparação qualitativa em relação às imagens obtidas, usando os operadores de migração pré-empilhamento Kirchhoff (K-PSDM) e Kirchhoff-feixes gaussianos (KGB-PSDM) em verdadeira amplitude 2,5D em profundidade no domínio $\mathrm{CO}$ com gradiente constante de velocidade, utilizando um dado sintético sob a forma de um anticlinal sem e com ruído aditivo aleatório. Será apresentada ainda, uma comparação entre as amplitudes extraídas dos processos acima citados, sem e com ruído aditivo aleatório, juntamente com os coeficientes de reflexão, os quais são oriundos da modelagem, onde o objetivo é destacar a estabilidade e a acurácia do algoritmo aqui proposto (KGB-PSDM) em relação ao algoritmo convencional (K-PSDM), no que concerne à correção do efeito do espalhamento geométrico.

\section{Meio com gradiente constante de velocidade}

Nesta situação foi utilizado o programa SEIS88 (Červený; Psenčick, 1988), através da teoria do raio de ordem zero para gerar um dado sísmico oriundo de um modelo que possui uma camada com gradiente vertical constante sobre um substrato homogêneo, com velocidade igual a $3500 \mathrm{~m} / \mathrm{s}$ separados por uma interface suave (Figura 2). A velocidade $v$ da camada superior varia com a profundidade $z$ de acordo com a expressão:

$$
v=v_{0}+g\left(z-z_{0}\right)
$$

onde, $g$ define o gradiente da velocidade, $z_{0} \quad 0$ valor da profundidade inicial e $\quad v_{0}$ a velocidade inicial. Neste contexto, o gradiente assumido possui um valor de $g=0.7 s^{-1}$ com $v_{0}$ igual a $2000 \mathrm{~m} / \mathrm{s}$. Também é mostrado na Figura (3) a seção sísmica de entrada com afastamento constante de $500 \mathrm{~m}$ com ruído aleatório aditivo, sendo neste último a razão sinal ruído igual a 3.

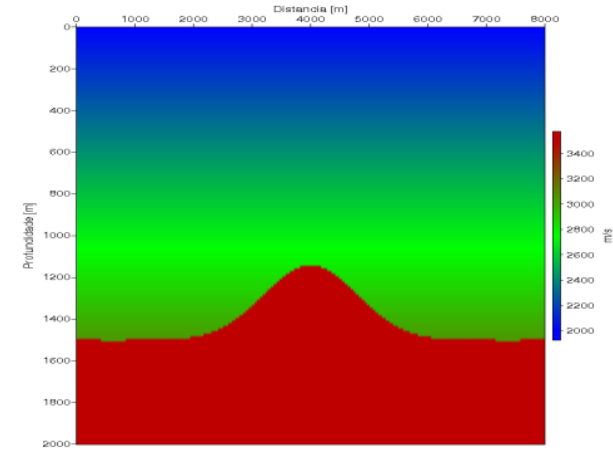

Figura 2- Modelo de velocidades com gradiente vertical constante $\left(g=0.7 \mathrm{~s}^{-1}\right)$, representando um refletor curvo sob a forma de um anticlinal.

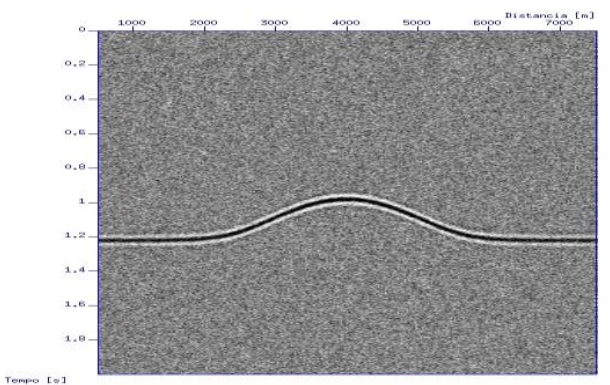

Figura 3- Seção sísmica sintética com afastamento constante $500 \mathrm{~m}$ (dado de entrada) para o teste de migração com ruído aleatório aditivo, onde a razão sinal ruído é igual a 3.

\section{Resposta ao impulso}

A Figura (4) mostra a resposta ao impulso do operador Kirchhoff convencional (esquerda) e a resposta ao impulso do operador de migração Kirchhoff modificado (direita), para um traço com afastamento $500 \mathrm{~m}$. Este último resultado foi obtido utilizando uma aproximação do campo de ondas, definida por uma integral de superposição de feixes gaussianos, onde a mesma foi inserida no núcleo do operador Kirchhoff convencional.
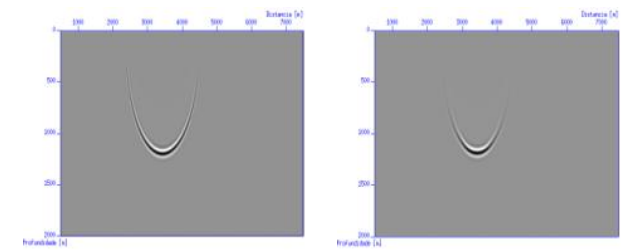

Figura 4- Respostas ao impulso para um traço com afastamento $500 \mathrm{~m}$. Esquerda: Kirchhoff convencional. Direita: Kirchhoff modificado.

\section{Migração K-PSDM e KGB-PSDM sem ruído}

Os resultados das migrações K-PSDM e KGB-PSDM representados pelas Figuras (5 e 6), respectivamente, 
apresentam o refletor em subsuperfície na sua verdadeira posição espacial, visto utilizar-se a verdadeira velocidade de migração. No entanto, a imagem migrada pelo método KGB-PSDM (Figura 6) apresenta-se com uma resolução maior do refletor, principalmente na região central do modelo, quando comparada com a imagem advinda do processo K-PSDM (Figura 5), ressaltando que os efeitos de bordas presentes em ambas as seções migradas são mais atenuados na Figura (6).

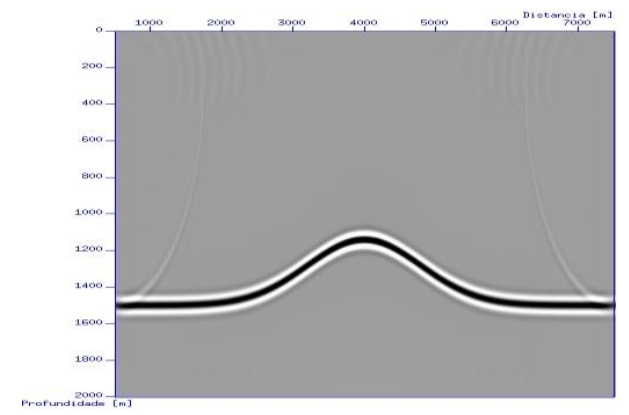

Figura 5- Migração Kirchhoff pré-empilhamento em profundidade (K-PSDM) sem a presença de ruído aleatório aditivo.

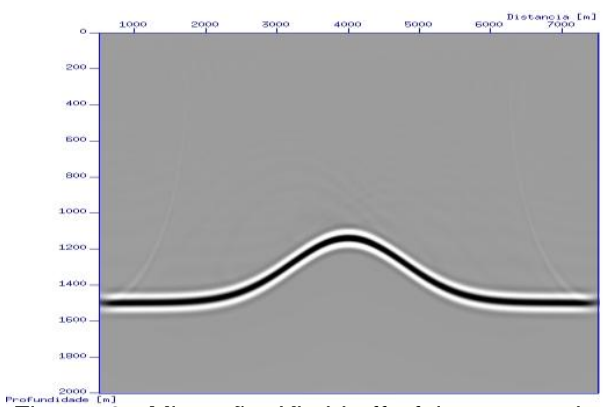

Figura 6- Migração Kirchhoff- feixes gaussianos préempilhamento em profundidade (KGB-PSDM) sem a presença de ruído aleatório aditivo.

\section{Migração K-PSDM e KGB-PSDM com ruído}

Adicionando ruído aleatório no dado de entrada, razão sinal ruído igual a 3 (Figura 3), foram aplicados os métodos K-PSDM e KGB-PSDM, cujos resultados são apresentados nas Figuras (7 e 8), respectivamente. As referidas imagens apresentam uma satisfatória continuidade no refletor, porém, as mesmas diferem quanto à resolução, uma vez que na Figura (7) nota-se uma contaminação maior de ruído quando comparada à Figura (8).

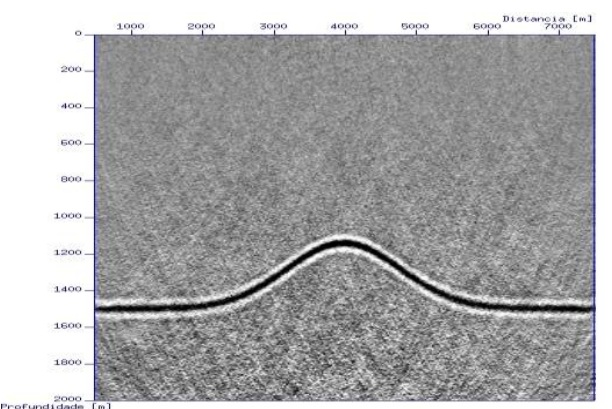

Figura 7- Migração Kirchhoff pré-empilhamento em profundidade (K-PSDM) com a presença de ruído aleatório aditivo, onde a razão sinal ruído é igual a 3.

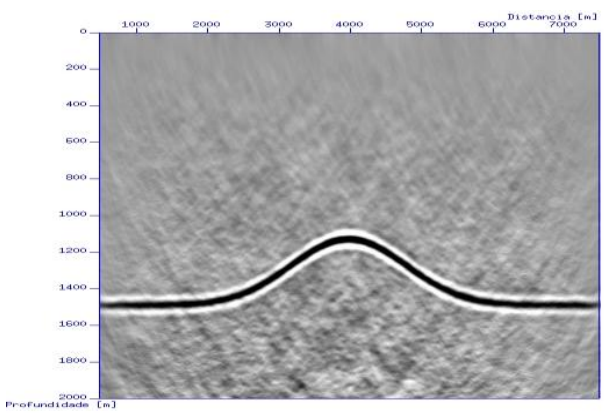

Figura 8- Migração Kirchhoff-feixes gaussianos préempilhamento em profundidade (KGB-PSDM) com a presença de ruído aleatório aditivo, onde a razão sinal ruído é igual a 3.

\section{Amplitudes verdadeiras (meio com gradiente constante de velocidade)}

Para verificar o conceito de amplitudes verdadeiras (Schleicher et al., 1993; Tygel et al., 1996), foram retiradas as amplitudes das seções migradas (estimativa do coeficiente de reflexão) e comparadas com o valor exato do coeficiente de reflexão oriundo da modelagem de acordo com a seguinte ordem:

I- $\quad$ Sem a presença de ruído aleatório aditivo (K-PSDM - KGB-PSDM - Coeficiente reflexão) (Figura 9);

II- $\quad$ Com a presença de ruído aleatório aditivo (razão sinal ruído igual a 15) (K-PSDM KGB-PSDM- Coeficiente reflexão) (Figura $10)$. 


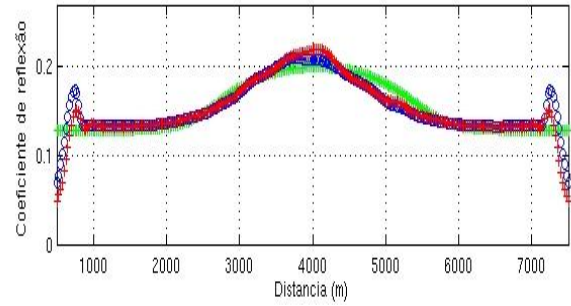

Figura 9- Coeficientes de reflexão sem a presença de ruído aleatório aditivo. Cor azul: obtido a partir da seção migrada pelo operador K-PSDM. Cor vermelha: obtido a partir da seção migrada pelo operador KGB-PSDM. Cor verde: valor exato.

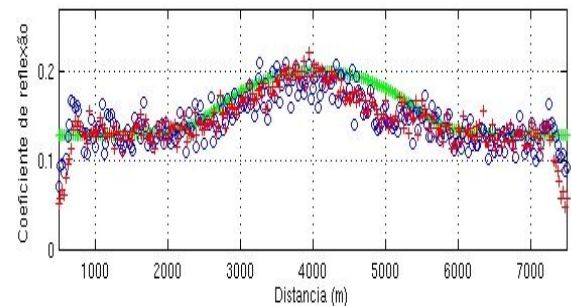

Figura 10- Coeficientes de reflexão com a presença de ruído aleatório aditivo, onde a razão sinal / ruído é igual a 15. Cor azul: obtido a partir da seção migrada pelo operador K-PSDM. Cor vermelha: obtido a partir da seção migrada pelo operador KGB-PSDM. Cor verde: valor exato.

\section{Conclusões}

Neste trabalho, introduzimos um procedimento de migração conhecido como Kirchhoff-feixes gaussianos, o referido procedimento é um operador integral, que trabalha no núcleo do operador de migração Kirchhoff convencional ao longo do dado sísmico, sobrepondo reflexões paraxiais presentes na vizinhança do traço de referência e restrita à zona de Fresnel projetada do experimento sísmico, o mesmo faz uso de duas funções pesos: uma para o núcleo da integral de Kirchhoff (integral externa) de maneira idêntica a descrita em Schleicher et al., (1993) e uma segunda para integral de superposição de feixes gaussianos (integral interna) a qual é proporcional a zona de Fresnel projetada, embora aqui definida de maneira completa ad hoc, assim o significado físico é baseado na óptica geométrica (raios), por outro lado, também é matematicamente semelhante as abordagens baseadas na equação da onda, por exemplo, Hill (1990) e Hill (2001). De maneira prática, tal operador pode ser visto como uma migração "slant stack" de eventos em torno de um traço de referência. A migração KGB-PSDM foi testada em um dado sísmico sintético com afastamento constante de $500 \mathrm{~m}$ sem e com a presença de ruído aleatório aditivo. Os resultados mostraram um aumento na resolução das imagens migradas quando comparadas com as imagens oriundas da utilização do algoritmo de migração K-PSDM. Em relação à atenuação do espalhamento geométrico, observou-se que tanto o algoritmo de migração Kirchhoff

e Kirchhoff-feixes gaussianos estimaram os coeficientes de reflexão com uma precisão aceitável, tanto para a situação em que o dado de entrada estava sem, como também com ruído aleatório aditivo. Portanto, baseandose nos resultados preliminares apresentados neste trabalho, o algoritmo Kirchhoff-feixes gaussianos mostrase uma ferramenta adicional sendo uma alternativa atraente para a interpretação e o imageamento sísmico, estimando amplitudes sísmicas como indicadores de hidrocarbonetos.

\section{Agradecimentos}

Agradecemos a Rede de Geofísica da Petrobras pelo apoio financeiro na qualidade de bolsa de pesquisa ao primeiro autor deste trabalho.

\section{Referências}

ABERTIN, U.; YINGST, D.; KITSCHENSIDE, P., 2004. True-amplitude beam migration. SEG Expanded Abstracts of the 74th. Annual Meeting, Denver, USA.

BLEISTEIN, N., 1987. On the imaging of reflectors in the Earth. Geophysics,52:931-942.

BLEISTEIN, N., 2008. Mathematics of modeling, migration and inversion with Gaussian beams beams. Monograph, CWP, Colorado, USA.

ČERVENÝ, V. 1982. Expansion of a plane wave Gaussian beans. Studia Geoph et Geod, v.26, p.120-131.

ČERVENÝ, V. 1983. Synthetic body wave seismograms for laterally varying layered structures by the Gaussian Beam method. Geophys. J. R. astr. Soc, v.73, p.389-426.

ČERVENÝ, V. 1985. Gaussian beam synthetic seismograms. J. Geophys., v.58, p.44-72.

ČERVENÝ, V; PŠENČíK, I., 1988. SEIS88, Ray tracing program package.

ČERVENÝ, V. 2000. Summation of paraxial Gaussian beams and of paraxial ray approximations in inhomogeneous anisotropic layered structures. Seismic waves in complex 3D structure, Report, Charles University, Prague, v.10, p. 121-159.

COSTA, M. J. S. 2012. Migração pré-empilhamento Kirchhoff feixes gaussianos 2,5D nos domínios afastamento comum e ângulo comum. Universidade Federal do Pará, Programa de Pós- Graduação em Geofísica, Belém, Pará. (Tese de Doutorado).

FERREIRA, C. A. S. 2007. Migração Kirchhoff préempilhamento em profundidade modificada usando o operador de feixes gaussianos. Universidade Federal do 
Pará, Programa de Pós- Graduação em Geofísica, Belém, Pará. (Tese de Doutorado).

FERREIRA, C. A. S.; CRUZ, J. C. R., 2004a. Modified Kirchhoff prestack migration using the Gaussian Beam operator as Green function. IN: EAGE CONFERENCE \& EXHIBITION, 66., Paris, France. CD-ROM.

FERREIRA, C. A. S.; CRUZ, J. C. R., 2005a. Migração pré-empilhamento em profundidade usando o operador de feixes gaussianos como função de Green - Resultados numéricos e teóricos. IN: WORKSHOP DA REDE NORTE DE RISCO EXPLORATÓRIO, 2., Belém. CDROM.

FERREIRA, C. A. S.; CRUZ, J. C. R., 2005b. Modified Kirchhoff prestack depth migration using the Gaussian beam operator as Green function - Theoretical and numerical results. Wave Inversion Technology. Karlsrhue, Alemanha. (Report 8).

FERREIRA, C. A. S.; CRUZ, J. C. R., 2005c. Modified Kirchhoff prestack depth migration using the Gaussian beam operator as Green function - Theoretical and numerical results. IN: INTERNATIONAL CONGRESS OF THE BRAZILIAN GEOPHYSICAL SOCIETY, 9., Salvador. CD-ROM.

FERREIRA, C. A. S.; CRUZ, J. C. R., 2005d. Migração KGB-PSDM em meios com gradiente constante de velocidade. IN: WORKSHOP DA REDE COOPERATIVA DE PESQUISA EM RISCO EXPLORAT'ORIO, 3., Salvador. CD-ROM.

FERREIRA, C. A. S.; CRUZ, J. C. R. A., 2009 Comparison of two true-amplitude Gaussian beam migration/inversion operators. In: INTERNATIONAL CONGRESS OF THE BRAZILIAN GEOPHYSICAL SOCIETY, 11, Salvador. Anais. Salvador: SBGf. CDROM.

GRAY, S. H.; BLEISTEIN, N., 2009. True-amplitude Gaussian-beam migration. Geophysics, v.74, n.2, s11s23.

HILL, N. R., 1990. Gaussian beam migration. Geophysics, v.55, p.1416-1428.

HILL, N. R., 2001. Prestack Gaussian beam depth migration. Geophysics, v.66, p.1240-1250.

KRAVTSOV Y. A.; BERCYNKI P., 2007. Gaussian Beams in inhomogeneous media: a review. Studia Geoph. et Geod, v.51, p.1-36.

MARTINS, J.M.; SCHLEICHER, J.; TYGEL, M.; SANTOS, L.T., 1997. True-amplitude migration and demigration. Journal of Seismic Exploration, v.6, p.159180.
MÜLLER, G. Efficient calculation of Gaussian beam seismograms for two dimensional inhomogeneous media., 1984. Geophysics. J. R. Astr. Soc, v.79, p.153166.

NOWACK, R. L. Calculation of synthetic seismograms with Gaussian beams., 2003. Pure and Applied Geophysics, v.160, p.487-507.

POPOV, M. M. A new method of computation of wave fields using Gaussian beams., 1982. Wave Motion, v.4, p.85-97.

POPOV, M. M., 2002. Ray theory and Gaussian beam method for geophysicists. EDUFBA, Salvador, Bahia, Brasil, 172p.

POPOV, M. M.; SEMTCHENOK, N. M.; POPOV, P. M., 2010. Depth migration by the Gaussian beam summation method. Geophysics, v.75, p.81-93.

PROTASOV, M. I.; TCHEVERDA, V. A., 2011. True amplitude imaging by inverse generalized Radon transform based on Gaussian beam decomposition of acoustic Green's function. Geophysical Prospecting, v.59, p.197-209.

SCHLEICHER, J.; TYGEL, M.; HUBRAL, P., 1993. 3D true - amplitude finite - offset migration, Geophysics, v.58, n.8, p.1112 - 1126 .

SCHNEIDER, J.; W. A., 1978. Integral formulation for migration in two and three dimensions. Geophysics, v.43, p.49-76.

TYGEL, M.; SCHLEICHER, J.; HUBRAL, P., 1996. An unified approach to $3 D$ seismic reflection imaging - Part II: Theory. Geophysics, v.61, p.759-775.

ŽÁČEK K., 2006. Optimization of the shape of Gaussian beams. Studia Geoph et Geod, v.50, p.349-365. 